$7-2013$

\title{
Reliability-based Topology Optimization of Trusses with Stochastic Stiffness
}

\author{
Mehdi Jalalpour \\ Cleveland State University, m.jalapour@csuohio.edu \\ James K. Guest \\ Johns Hopkins University \\ Takeru Igusa \\ Johns Hopkins University
}

Follow this and additional works at: https://engagedscholarship.csuohio.edu/encee_facpub

Part of the Structural Engineering Commons

How does access to this work benefit you? Let us know!

\section{Publisher's Statement}

NOTICE: this is the author's version of a work that was accepted for publication in Structural Safety. Changes resulting from the publishing process, such as peer review, editing, corrections, structural formatting, and other quality control mechanisms may not be reflected in this document. Changes may have been made to this work since it was submitted for publication. A definitive version was subsequently published in Structural Safety, 43, (July 2013) DOI:10.1016/ j.strusafe.2013.02.003

\section{Original Citation}

Jalalpour, M., Guest, J., and Igusa, T., "Reliability-based topology optimization of trusses with stochastic stiffness”, Structural Safety 43: 41-49, 2013

This Article is brought to you for free and open access by the Civil and Environmental Engineering at EngagedScholarship@CSU. It has been accepted for inclusion in Civil and Environmental Engineering Faculty Publications by an authorized administrator of EngagedScholarship@CSU. For more information, please contact library.es@csuohio.edu. 


\title{
Reliability-based topology optimization of trusses with stochastic stiffness
}

\author{
Mehdi Jalalpour*, James K. Guest, Takeru Igusa \\ Department of Civil Engineering, Johns Hopkins University Baltimore, MD 21218, USA
}

Post-print prepared by MSL Academic Endeavors, the imprint of the Michael Schwartz Library at Cleveland State University, 2014

\section{Introduction}

Topology optimization is a computational design tool for optimizing structural system connectivity and corresponding member sizes to meet given performance targets. Due to associated numerical challenges, however, the vast majority of works consider the loading and structural characteristics, such as geometry and material properties, to be deterministic. This is almost always an unrealistic assumption due to the inherent variability in manufacturing process and simplifying assumptions for evaluating the loads on structure. Formal design methods accounting for such uncertainties include worst-case design, robust topology optimization (RTO), and reliability-based topology optimization (RBTO). This work focuses on RBTO of truss structural systems with stochastic stiffness.

The goal of RBTO is to find the optimal values for a design vector $\boldsymbol{\rho}$ so that a target reliability is achieved. Randomness in the system is expressed as basic variables and denoted by $\boldsymbol{\eta}$. It is useful to define an auxiliary function $s(\boldsymbol{\eta}, \boldsymbol{\rho})$ for the load effects, which can be displacement, stress, etc. Failure is defined by a limit-state function in terms of load effects as $g(s)$. Probability of failure can be found in terms of $s$ by

$P_{\text {fail }}=\int_{\mathrm{d}(s)<0} f_{s}(s) d s$

where $f_{s}(s)$ is the probability distribution of load effects. It is apparent that information regarding the distribution of the load effects and form of the limit-state function is needed to calculate the above inte-

\footnotetext{
* Corresponding author.

E-mail address: mehdi@jhu.edu (M. Jalalpour).
}

gral directly. Such an approach is called Level III reliability analysis [1]. However, only the distribution of basic variables $\boldsymbol{\eta}$ is typically known, making assessment of the probability of failure a challenging task. Moreover, in the engineering cases where there may be many uncertain parameters, calculation of the integral might become computationally infeasible [2]. To circumvent these issues, Level II reliability methods have been proposed, where the notion of reliability index $[3,4]$ is used. To follow this methodology, one first maps the basic variables to the space of standard normal variables using a probabilistic transformation. The limit surface is then approximated by a first (FORM) or second (SORM) order Taylor series [5] and the normal distribution may be used to estimate the structural reliability (see Hohenbichler et al. [6], Gasser and Schueller [7] for a review). The key idea is that the normal probability density function (PDF) decays very quickly with distance from the origin, limiting significant contributions to the response integral in Eq. (1) to points in the neighborhood of the most probable point (MPP) with the least distance to the origin $(\beta)$. Therefore, a Taylor series expansion about this point leads to acceptable reliability estimates, and the problem is now transformed to finding the MPP. There are a number of search methods for optimal points and we refer to [8] for a review on the available methods. It is then possible to find the probability of failure. For instance, FORM utilizes $P_{\text {fail }}=\Phi(-\beta)$, where $\Phi($.) symbolizes the normal cumulative distribution function (CDF).

In the context of topology optimization, the MPP must be located at every iteration of the optimization process. This produces a nested, or double-loop optimization algorithm $[9,10]$, which may ultimately lead to cumbersome or even intractable computations. Tu et al. [11] report that using the Hasofer-Lind definition for the reliability index may lead to convergence issues on locating 
the MPP and that solutions may not exist. Methods for avoiding the double-loop approach have also been proposed [12,13]. Often, these methods consist of employing the Karush-Kuhn-Tucker (KKT) optimality conditions for an approximation to the design point in the nested loop, allowing the inner loop to be replaced with a deterministic constraint [14]. Kharmanda et al. [15] have proposed a decoupling method where they first search for the MPP based on the desired reliability of design, and then perform a deterministic minimum compliance design. Du and Chen [16] have proposed an interesting methodology to replace the search for the design point by the method of sequential optimization and reliability assessment. Kang and Luo [17] have implemented a non-probabilistic method to perform reliability-based design on geometric nonlinear continuum structures using a convex model that encompasses all the possible values for basic random variables. We refer to [18] for a review on the methodologies on singleor nested-loop optimization.

The works in the preceding discussion can be categorized as component-level reliability. When considering system reliability, multiple performance functions must be considered in either series or parallel, significantly increasing computational cost $[19,20]$. ThoftChristensen and Dalsgård Sørensen [21] proposed a methodology to evaluate structural reliability with correlated member uncertainties for both parallel and series systems. Nguyen et al. [22] have generalized the methodology proposed in [14] to system reliability. Silva et al. [23] have used a FORM single-loop approach for reliability-based design on both component and system level for the case when uncertainty is present in loading. Their methodology utilizes an approximation to MPP and the gradient, and is suitable for integration with available finite-element software. Park et al. [24] have proposed an efficient methodology for computing system reliability indices in terms of component reliability. Their methodology is based on considering correlation between modes of failure.

There is a rich literature on various applications of reliabilitybased design, and the reader is referred to Tsompanakis et al. [25] for a recent collection of state-of-the-art papers, Schueller and Jensen [26] for an excellent review on the recent advancements on design under uncertainty, and Igusa and Der Kiureghian [27] for a review in dynamics problems and the importance of considering randomness in structural characteristics. Specifically in the context of structural topology optimization, a significant amount of work has considered RBTO under uncertainty in loading for structures defined on truss [28] and continuum [29] domains.

An emerging trend in topology optimization has been the formal consideration of uncertainty in structural characteristics. This has largely fallen under the regime of robust topology optimization, i.e., design for insensitivity to variations in manufactured stiffness. The standard measure of structural response is the compliance, defined as $c=\mathbf{f}^{T} \mathbf{d}$, which is a proxy for structural stiffness. Guest and Igusa [30] used a perturbation-based approach to minimize the expected value of compliance under geometric uncertainties. This work was later extended to include uncertainties in material properties [31], more general robust design optimization [32], and global instability effects [33]. Chen et al. [34] used Karhunen-Loeve expansion and multivariate dimension reduction to minimize the expected value of compliance under uncertainty in material properties as well as applied loads. Schevenels et al. [35] use Monte Carlo simulation to formally account for over- and under-etching in manufacturing to optimize the expected value of response. Tootkaboni et al. [36] use polynomial chaos to minimize the expected value and variability of stiffness under material property uncertainties.

In the present article, it is shown that the probability distribution of the compliance of optimally designed trusses with uncertain nodal locations and/or members Young's modulus can be approximated by the Gumbel distribution, provided that the truss is not a trivial structure with only a few nodes. Because of this ob- served property the probability of failure can be readily estimated from first- and second-moments of the response, circumventing the need for a nested-loop approach. This enables straightforward expressions for sensitivity analysis and produces an effective and computationally efficient topology optimization design algorithm, especially in system-level RBTO, and ultimately allows the designer to perform RBTO at the computational cost of RTO.

This article is structured as follows. We begin by formulating the limit-state function and structural reliability in terms of a displacement-based metric. We then derive the expressions for the statistical moments of the metric using stochastic perturbations and show how these moments are related to the parameters of the Gumbel distribution. We then propose a single-loop reliability-based topology optimization method for truss systems. Numerical examples are presented for trusses with geometric and material property uncertainties and the accuracy of the method is verified using Monte Carlo simulation.

\section{Reliability-based topology optimization formulation}

In this section, we begin with defining the structural reliability in terms of a displacement-based metric, obtain the required statistics of the metric, and present the reliability-based topology optimization (RBTO) formulation.

\subsection{Structural reliability}

To assess the structural reliability, structural performance has to be measured in terms of a load effect (stress, deflection,...), and a limit-state or performance function is defined for the structure. We measure structural performance in terms of a linear function of displacement vector using:

$c=\mathbf{l}^{T} \mathbf{d}$

where $\mathbf{l}$ is a vector that linearly combines the degrees of freedom of interest from the displacement vector $\mathbf{d}$. Throughout this article boldface lower and upper case letters represent vectors and matrices, respectively. We can constrain deflection at degree of freedom $i$ by using $\mathbf{l}=\mathbf{e}_{i}$, where $\mathbf{e}_{i}$ is a unit vector with zeros at all degrees of freedom other than $i$. We can now define the limit-state function in terms of the displacement metric as

$g=c^{*}-c$

where $c^{*}$ is the predetermined structure capacity. For example, under a deflection constraint $\left(\mathbf{l}=\mathbf{e}_{i}\right), c^{*}$ represents the maximum allowable deflection (capacity) at degree of freedom $i$ and $c$ represents the corresponding actual (demand) deflection. This problem formulation is natural, as typically design requires that, for instance, the structure displacement be less than a given deterministic value (e.g., 1/360 of span). The probability of failure can be evaluated using:

$P_{\text {fail }}=P[g<0]=P\left[c^{*}<c\right]=1-P\left[c \leq c^{*}\right] \leftarrow$

There are now two challenges in using the above formulations: obtaining the displacement metric and obtaining its distribution. These challenges and possible mitigation strategies are discussed in further detail in the remaining parts of this section.

\subsection{Structural response}

When uncertainty is introduced into structural stiffness, such as in the form of geometric imperfections or material variability, obtaining structural displacement becomes challenging, because it is related to the inverse of a stochastic matrix. In this subsection, we review a perturbation approach to this problem that closely follows Guest and Igusa [30]. First we express each random quantity as: 
$\eta_{i}=\eta_{i 0}+\delta \eta_{i}$

$(5) \leftarrow$

where $\eta_{i 0}$ is the deterministic and $\delta \eta_{i}$ is the additive random component. Information about the distribution of $\delta \boldsymbol{\eta}$ and its first and second statistical moments are assumed known (e.g., through measurement). Without loss of generality we may assume that $\delta \boldsymbol{\eta}$ has zero mean. Structural equilibrium is given as:

$\mathbf{f}=\mathbf{K}(\eta) \mathbf{d}(\eta) \leftarrow$

where the applied loads $\mathbf{f}$ are assumed deterministic. It is observed that the input variability in structural stiffness makes the displacement a random vector. Because the uncertainties of interest are due to manufacturing defects or material variability, it assumed that these uncertainties are relatively small. This enables the displacement for any structure with stochastic response to be obtained using perturbations of the stiffness matrix to the second order, yielding:

$$
\begin{aligned}
\mathbf{d}= & \mathbf{K}_{0}^{-1} \mathbf{f}-\mathbf{K}_{0}^{-1} \mathbf{K}_{0, i} \mathbf{K}_{0}^{-1} \mathbf{f} \delta \eta_{i} \\
& +\mathbf{K}_{0}^{-1} \mathbf{K}_{0, i} \mathbf{K}_{0}^{-1} \mathbf{K}_{0, j} \mathbf{K}_{0}^{-1} \mathbf{f} \delta \eta_{i} \delta \eta_{j} \\
& -\frac{1}{2} \mathbf{K}_{0}^{-1} \mathbf{K}_{0, j} \mathbf{K}_{0}^{-1} \mathbf{f} \delta \eta_{i} \delta \eta_{j}
\end{aligned}
$$

where we have used standard indicial notation with repeated index meaning summation. The matrix $\mathbf{K}_{0, \mathrm{i}}$ denotes the derivative of the deterministic stiffness matrix with respect to the $i$ th basic variable. To obtain the displacement metric, let us rewrite (7) in vector format to produce a more compact form. We first express the randomness as:

$\mathbf{x}=\mathbf{e}_{i} \delta \eta_{i}$

and denote the solution of the following adjoint problem as $\hat{\mathbf{d}_{0}}$ :

$\mathbf{K} \hat{\mathbf{d}_{0}}=\mathbf{l}$

It is common practice in topology optimization to use $\mathbf{l}=\mathbf{f}$, making $c$ proportional to the external work, or compliance, which can be interpreted as a proxy for structural stiffness. For this case $\hat{\mathbf{d}}_{0}=\mathbf{d}_{0}$, which is the deterministic solution under applied loads. However, let us continue the derivations with the general case. Substituting (9) and (7) into (2) gives:

$c=\mathbf{l}^{T} \mathbf{d}_{0}-\hat{\mathbf{d}}_{0}^{T} \mathbf{K}_{0, i} \mathbf{d}_{0} \mathbf{e}_{i}^{T} \mathbf{x}+\mathbf{x}^{T} \mathbf{e}_{i} \hat{\mathbf{d}}_{0}^{T} \mathbf{K}_{0, i} \mathbf{K}_{0}^{-1} \mathbf{K}_{0, j} \mathbf{d}_{0} \mathbf{e}_{j}^{T} \mathbf{x}$

$-\frac{1}{2} \mathbf{x}^{T} \mathbf{e}_{i} \hat{\mathbf{d}}_{0}^{T} \mathbf{K}_{0, i j} \mathbf{d}_{0} \mathbf{e}_{j}^{T} \mathbf{x}$

Observe that the random displacement-related performance metric $c$ is now written in terms of the deterministic stiffness matrix of structure and its derivatives, which are straightforward to compute [30]. Therefore, under the assumption of small uncertainties, we can evaluate the structural response using Eq. (10) for any structural system. To rewrite the above in a more compact format, let us define $\mathbf{U}$ and $\hat{\mathbf{U}}$ as dimensionless matrices that can be understood as the structural normalized displacements under a system of equivalent normalized loads [33] as follows:

$\mathbf{U}=\mathbf{K}_{0}^{-1} \mathbf{F}$

$\hat{\mathbf{U}}=\mathbf{K}_{0}^{-1} \hat{\mathbf{F}}$

where the system of equivalent normalized loads $\mathbf{F}$ and $\hat{\mathbf{F}}$ can be defined as:

$\mathbf{F}=-\mathbf{K}_{0, i} \mathbf{d}_{0} \mathbf{e}_{i}^{T}$

$\hat{\mathbf{F}}=-\mathbf{K}_{0, i} \hat{\mathbf{d}}_{0} \mathbf{e}_{i}^{T}$

Substituting (11) into (10) yields:

$c=\mathbf{l}^{T} \mathbf{d}_{0}+\hat{\mathbf{d}}_{0}^{T} \mathbf{K}_{0} \mathbf{U} \mathbf{x}+\mathbf{x}^{T} \hat{\mathbf{U}}^{T} \mathbf{K}_{0} \mathbf{U} \mathbf{x}-\frac{1}{2} \mathbf{x}^{T} \mathbf{e}_{i} \hat{\mathbf{d}}_{0}^{T} \mathbf{K}_{0, j} \mathbf{d}_{0} \mathbf{e}_{j}^{T} \mathbf{x}$

We can transform (13) into a more compact form by defining:
$\mathbf{K}_{\mathrm{U}}=\hat{\mathbf{U}}^{T} \mathbf{K}_{0} \mathbf{U}-\frac{1}{2} \mathbf{e}_{i} \hat{\mathbf{d}}_{0}^{T} \mathbf{K}_{0, i j} \mathbf{d}_{0} \mathbf{e}_{j}^{T}$

It is noted that $\mathbf{K}_{\mathrm{U}}$ is symmetric due to symmetry of the stiffness matrix. Substituting (14) into (13) yields the final form for the displacement metric as:

$c=\mathbf{l}^{T} \mathbf{d}_{0}+\hat{\mathbf{d}}_{0}^{T} \mathbf{K}_{0} \mathbf{U} \mathbf{x}+\mathbf{x}^{T} \mathbf{K}_{\mathbf{U}} \mathbf{x}$

Two types of uncertainties are considered in this work: geometric imperfections and material variability. Expressions for the derivatives $\mathbf{K}_{0, \mathrm{i}}$ and $\mathbf{K}_{0, \mathrm{ij}}$ for geometric uncertainties are available in [30] and are straightforward for trusses. They are likewise straightforward for uncertainty in Young's modulus [32,36], where in particular we note:

$\mathbf{K}_{0, i j}=0 \quad \forall i, j$

Eq. (15) is general and can be used to obtain the displacement metric for any source of uncertainty and for all structural systems.

\subsection{Gumbel distribution}

We can view the displacement-based metric as a mechanical transformation of the input variability in structural characteristics. Now depending on the form of this transformation, the metric might have various distributions, and obtaining them is not a trivial task. It has been shown that FORM and SORM methods perform very well in estimating the probability of failure $[5-7,37]$. However, as discussed in the introduction, these methods may become computationally prohibitive in the context of the iterative structural topology optimization.

One approach to overcome this difficulty is to look for an appropriate distribution to accurately model the displacement metric. We used Liu and Der Kiureghian [38], which lists the most widely used two-parameter distributions for reliability analysis (Normal, Lognormal, Weibull, Shifted exponential and Gumbel) and observed that Gumbel distribution provides, by far, the closest fit to the empirical distributions of the demand metric in Eq. (2) for all non-trivial trusses when the basic variables are normally distributed. It is noted that using the normal distribution for the demand is inappropriate because of the quadratic terms in $\mathbf{x}$ in Eq. (13).

Gumbel, or extreme type I, is a two parameter distribution dependent on location and scale variables, denoted by $l$ and $s$, respectively. The cumulative distribution function (CDF) is given by [39]:

$F_{C}\left(c^{*}\right)=\exp \left(\left(-\exp \left(-\left(\frac{c^{*}-l}{s}\right)\right)\right)\right.$

Assuming that the displacement metric $c$ follows this distribution, we can establish a relation between the distribution parameters and demand using the method of moments:

$s=\frac{\sqrt{6} \phi_{c}}{\pi}$

$l=\mu_{c}-\gamma s$

where $\gamma$ is the Euler-Mascheroni constant, and $\mu_{c}$ and $\sigma_{c}$ are the expected value and standard deviation of $c$, respectively. Combining Eqs. (4) and (17) and following Ditlevsen [40], we can express the reliability index as:

$\beta=\Phi^{-1}\left(F_{C}(\lambda)\right) \leftarrow$

where we have defined:

$\lambda=\stackrel{c^{* \leftarrow}-l}{s}$ 
Computation of the reliability index requires estimation of the expected value and standard deviation of the displacement-based metric (demand). These statistics are estimated using perturbation expansions and are derived in the next subsection.

\subsection{Response statistics}

Equations for estimating the expected value and standard deviation of compliance under stochastic stiffness have previously been derived using perturbation in Guest and Igusa [30], Asadpoure et al. [32], and Jalalpour et al. [33]. Herein, we extend these results for the more general displacement-based metric in (15). Following Jalalpour et al. [33], we can write the expected value of (15) as:

$E[c]=\mathbf{l}^{T} \mathbf{d}_{0}+\operatorname{tr}\left\{\mathbf{K}_{\mathrm{U}} \mathbf{C}_{0}\right\}$

where $\mathbf{C}_{0}$ is the covariance matrix of the basic random variables and $t r$ is the trace operator. Eq. (22) is general and does not depend on the distribution of basic variables.

It is useful to derive the demand variance for a general covariance matrix of normally distributed basic variables. The variance can be expressed as:

$\operatorname{var}[\mathbf{c}]=\operatorname{var}\left[\hat{\mathbf{d}}_{0}^{\mathrm{T}} \mathbf{K}_{0} \mathbf{U x}\right]+\operatorname{var}\left[\mathbf{x}^{\mathrm{T}} \mathbf{K}_{\mathrm{U}} \mathbf{x}\right] \leftarrow$

We note that the terms involving 3rd order statistics have been dropped because they are zero for normally distributed variables. The variance of the first term in (23) is straightforward to derive and is given as:

$\operatorname{var}\left[\hat{\mathbf{d}}_{0}^{T} \mathbf{K}_{0} \mathbf{U x}\right]=\hat{\mathbf{d}}_{0}^{T} \mathbf{K}_{0} \mathbf{U} \mathbf{C}_{0} \mathbf{U}^{T} \mathbf{K}_{0} \hat{\mathbf{d}}_{0}$

The variance for the second term of Eq. (23) is given as:

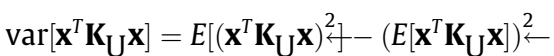

Using the trace operator we can derive the second term of Eq. (25) using:

$\left(E\left[\mathbf{x}^{T} \mathbf{K}_{\mathrm{U}} \mathbf{x}\right]\right)^{2} \longleftarrow\left(\operatorname{tr}\left\{\mathbf{K}_{\mathrm{U}} E\left[\mathbf{x} \mathbf{x}^{T}\right]\right\}\right)^{2} \longleftarrow \operatorname{tr}\left\{\mathbf{K}_{\mathrm{U}} \mathbf{C}_{0}\right\} \operatorname{tr}\left\{\mathbf{K}_{\mathrm{U}} \mathbf{C}_{0}\right\} \leftarrow$

To derive the variance of the first term of Eq. (25), we use Isserlis' theorem and indicial notation to get the 4th order statistics as:

$E\left[\mathbf{x}^{T} \mathbf{K}_{\mathrm{U}} \mathbf{X x}^{T} \mathbf{K}_{\mathrm{U}} \mathbf{x}\right]=k_{i j} k_{r s} E\left[x_{i} x_{j} x_{r} x_{s}\right] \leftarrow$

$=\sigma_{i j} k_{i j} \sigma_{r s} k_{r s}+\sigma_{i r} k_{i j} \sigma_{j s} k_{r s}+\sigma_{i s} k_{i j} \sigma_{j r} k_{r s}$

$=\sigma_{i j} k_{i j} \sigma_{r s} k_{r s}+2 \sigma_{i r} k_{i j} \sigma_{j s} k_{r s}$

where we have taken advantage of the symmetry of the matrices. Eq. (27) is now expressed using vector form and trace operator as:

$E\left[\mathbf{x}^{T} \mathbf{K}_{U} \mathbf{x} \mathbf{x}^{T} \mathbf{K}_{\mathbf{U}} \mathbf{x}\right]=\operatorname{tr}\left\{\mathbf{K}_{\mathbf{U}} \mathbf{C}_{0}\right\} \operatorname{tr}\left\{\mathbf{K}_{\mathrm{U}} \mathbf{C}_{0}\right\} \leftarrow$

$+2 \operatorname{tr}\left\{\mathbf{K}_{\mathrm{U}} \mathbf{C}_{0} \mathbf{K}_{\mathrm{U}} \mathbf{C}_{0}\right\}$

Substituting Eqs. (28) and (26) into Eq. (25) yields:

$\operatorname{var}\left[\mathbf{x}^{T} \mathbf{K}_{\mathrm{U}} \mathbf{x}\right]=2 \operatorname{tr}\left\{\left(\mathbf{K}_{\mathrm{U}} \mathbf{C}_{0}\right)^{2}\right\}$

Substituting Eqs. (24) and (29) into Eq. (23) gives the final form of variance as:

$\operatorname{var}[c]=\mathbf{l}^{T} \mathbf{U C}_{0} \mathbf{U}^{T} \mathbf{1}+2 \operatorname{tr}\left\{\left(\mathbf{K}_{\mathrm{U}} \mathbf{C}_{0}\right)^{2}\right\}$

where we have used the equilibrium condition of the adjoint problem defined in Eq. (9). It is noted that for uncorrelated basic variables, $\mathbf{C}_{0}$ would be a diagonal matrix. The above estimate for the variance includes terms up to 4th order statistics as noted in Eq. (27).

For cases where the basic random variables are not normally distributed Eq. (30) would no longer hold and the expression for the variance would have to be truncated to include only the second-order moments, as given by the first term in Eq. (30).

\subsection{Optimization formulation}

Using the above, we now present the reliability-based topology optimization formulation that minimizes structural volume $V$ (or equivalently weight) subject to a constraint on the probability of failure. This is formulated as a constraint on the reliability index as follows:

$$
\begin{aligned}
& \min V=\boldsymbol{\rho}^{T} \mathbf{v} \\
& \text { s.t. } \mathbf{K}_{0}(\boldsymbol{\rho}) \mathbf{d}(\boldsymbol{\rho})=\mathbf{f} \\
& \mathbf{K}_{0}(\boldsymbol{\rho}) \mathbf{U}(\boldsymbol{\rho})=\mathbf{F}(\boldsymbol{\rho}) \leftarrow \\
& \beta(\boldsymbol{\rho}) \geq \beta_{t} \\
& 0<\rho_{\min }<\rho^{e}
\end{aligned}
$$

where $\rho$ is the vector containing independent design variables $\left(\rho^{e}\right)$ and $\mathbf{v}$ is the vector of element volumes for unit $\rho$. For truss systems, these vectors are cross-sectional areas and element lengths respectively. Variable $\beta$ is the reliability index that has to exceed a target reliability $\beta_{t}$ (or equivalently the probability of failure has to be lower than a prescribed accepted risk). The lower design variable bound $\rho_{\min }$ is set to a small positive number to avoid singularity of the global stiffness matrix. The remaining constraints are for satisfying the equilibrium of the deterministic structure (with stiffness matrix $\mathbf{K}_{0}$ ) under the applied loads $\mathbf{f}$ and equivalent random loads $\mathbf{F}$. Consistent units are used throughout, hence all magnitudes are presented as unitless.

Problem (31) is continuous and thus is solved using gradientbased optimizers, selected herein as sequential quadratic programming as implemented in the MATLAB optimization toolbox [41]. Design sensitivities are derived using the direct differentiation method [33]. Expressions for these sensitivities are straightforward to derive, and details are presented in the appendix.

The proposed RBTO algorithm for stochastic stiffness offers computational efficiency in comparison to other MPP-based or Monte Carlo-based methods. The key advantages are: (1) using perturbation of the stiffness matrix the stochastic stiffness problem is recast in terms of equivalent normalized loads, meaning the linear systems to be solved feature one left-hand side ( $\mathbf{K}_{0}$ in Eq. (31)) with multiple right-hand sides (f and F in Eq. (31)), and (2) the Gumbel approximation eliminates the need to solve the inner MPP problem. Computational savings associated with advantage (1) were discussed in [30] and quantified in [32]. In short, the number of operations associated with evaluating demand statistical moments using perturbation is on the order of $n_{p}(O)=n_{e q}^{3} / 3+n_{r v} * n_{e q}^{2}$ and using Monte Carlo simulation is $n_{M C}(O)=M * n_{e q}^{3} / 3$, where $n_{e q}$ is the number of equations to solve (free degrees of freedom), $n_{r v}$ is the number of random variables, and $M$ is the number of Monte Carlo realizations needed to accurately quantify the demand variability. As $M$ is typically much larger than $n_{r v}$, the computational savings of using perturbation over Monte Carlo simulation are significant. With regard to the second advantage, double-loop algorithms use FORM or SORM which requires another minimization loop to estimate the reliability index at every design iteration. Assuming that this inner loop converges in $F$ iterations, it is straightforward to show that the total computational savings of the proposed algorithm over Monte Carlo-based FORM (or SORM) are on the order of $(F-1) * n_{M C}(O) / n_{p}(O)$. This is typically two or three orders of magnitude difference for the problems considered in this manuscript. More fundamentally regarding advantage (2), the estimates of structural reliability in Sections 2.3 and 2.4 enable us to achieve RBTO at the computational cost of RTO.

\section{Numerical examples}

The proposed algorithm is used to design several truss structures with uncertain nodal locations (geometric uncertainties) 


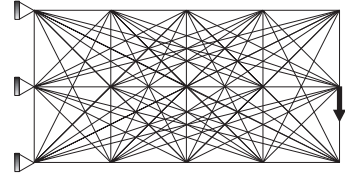

(a)

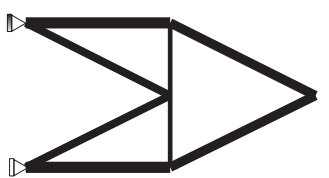

(b)
Fig. 1. Ground structure and deterministic design for cantilever truss - symmetric loading. (a) Ground structure and (b) deterministic design.

and uncertain Young's modulus (material uncertainties). The well-known ground structure approach to topology optimization is used [42-44]. In this approach, the design domain is densely meshed with candidate truss elements and material is distributed uniformly across the elements, which is the initial guess for the structural design. The proposed algorithm then optimizes cross-sectional areas throughout this ground structure. Inefficient (low-area) members are then removed and the optimization is re-run to 'clean-up' the result. This second optimization typically requires only a few iterations and does not lead to significant topological changes. In all cases, the random (basic) variables are Gaussian. For the examples with geometric uncertainties, the randomly located nodes associated with free degrees of freedom have standard deviations that are proportional to the mean spacing between nodes in the horizontal direction. For the example with material property uncertainties, the Young's modulus of each truss element is random with the same standard deviation from the deterministic modulus. The coefficient of variation for material uncertainty is 0.2 so the likelihood of negative Young's modulus is insignificant for the ranges of reliability of interest herein. Monte Carlo (MC) simulation using 10,000 realizations is used to verify the accuracy of the probability of failure estimates in all presented examples.

\subsection{Geometric uncertainty examples}

\subsubsection{Cantilever truss - symmetric loading}

We begin with a cantilever truss example to illustrate the direct effect of topology optimization using target reliabilities on design. The ground structure is shown in Fig. 1 (a). The dimensions are $60 \times 30$. This example has been widely studied in topology optimization literature using continuum, frame, or truss structures. For instance, Mogami et al. [45] studied this with beam elements under random loads using FORM. We consider geometric uncertainties of $8 \%$ nodal location variability, with a deterministic load of magnitude 0.25 .

We begin with deterministic design conditions and the standard minimum compliance problem meaning $\mathbf{l}=\mathbf{f}$ in Eq. (15). The minimum compliance solution under a total available volume constraint of $V_{\text {allow }}=900$ is shown in Fig. 1 (b). This design serves as our baseline for the subsequent discussion in this example. The compliance for this design is 3.52, which is now chosen as the capacity for the RBTO $\left(c^{*}=3.52\right)$, and the volume of the optimized designs will be normalized against $V_{\text {allow. }}$. When randomness is introduced to the nodal locations, a larger volume of material will be needed so that the probability of exceeding $c^{*}$ meets pre-assigned target reliabilities $\beta_{t}$. This volume will increase as the probability of exceedance decreases (and the corresponding target reliability increases).

Designs using formulation (31) that optimally satisfy three different values of target reliability, are shown in Fig. 2. It can be seen that material is added to the truss in two manners: (1) increased member sizing, where cross-sectional areas are increased (shown by line thickness in all design figures), and (2) changes in system topology, where new members are added to diversify the load path. Interestingly, no topological changes were observed from $\beta_{t}$ $=2.66$ to $\beta_{t}=3.01$.

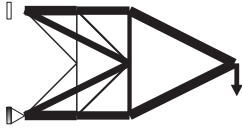

(a)

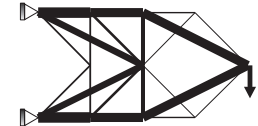

(b)

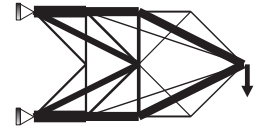

(c)
Fig. 2. Optimal cantilever truss designs for various values of target reliability. (a) $\beta_{t}$ $=1.46$, (b) $\beta_{t}=2.29$ and (c) $\beta_{t}=3.01$.

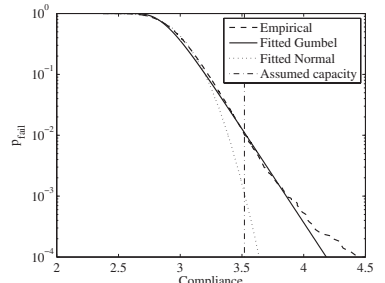

(a)

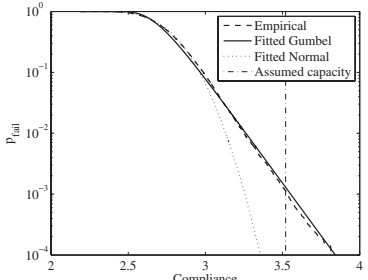

(b)
Fig. 3. Comparison between Gumbel and normal distribution fit for the designs in Fig. 2(b) and (c). (a) Design in Fig. 2(b), and (b) design in Fig. 2(c).

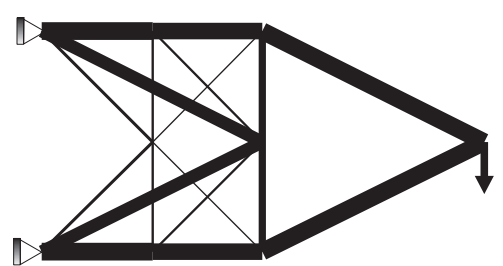

Fig. 4. Minimum expected value design for cantilever truss with $8 \%$ node location variability.

The accuracy of the estimates is verified using Monte Carlo simulation. Table 1 summarizes the results of this study. It is seen that the perturbation estimates of expected value and standard deviation are accurate. The table further confirms the assertion that the Gumbel distribution accurately captures behavior at the tails of the response distribution, even for low probabilities of failure.

In Table 1, the first column is normalized volume of each design, normalized by dividing by the volume of the deterministic design. This illustrates material cost associated with reduced probability of failure. It is noted that even in cases when topology does not change $\left(\beta_{t} \geq 2.66\right)$, member sizes increases, leading to larger total volume.

We examine the accuracy of the Gumbel prediction for $P_{\text {fail }}$ by plotting the empirical, Gumbel, and normal estimates on probabilities of failure with respect to the compliance capacity $c^{*}$. The probabilities for the designs in Fig. 2 (b) and (c) are shown in Fig. 3, which indicates that the Gumbel distribution closely follows the right tails of the Monte Carlo results. The plot also indicates that using a normal distribution for the demand would significantly underestimate the probability of failure.

We now compare these RBTO solutions to the solution found using robust design when considering only expected value of compliance, as originally presented in Guest and Igusa [30]. To make this comparison, we use the volume in the design of Fig. 2 (c) as the volume constraint and perform optimization to find the design with the minimum expected compliance (RTO). The result is shown in Fig. 4. While the structures in Figs. 2 (c) and 4 contain the same volume of material, the former which is optimized with respect to reliability has a more complex topology and is more redundant than the latter which is optimized with respect to expected compliance.

To compare the performance of RTO and RBTO, we conducted Monte Carlo simulations and plotted the corresponding PDFs in Fig. 5. As expected, the RTO solution has a lower expected value 
Table 1

Comparison summary between the proposed methodology and Monte Carlo simulation.

\begin{tabular}{|c|c|c|c|c|c|c|c|c|}
\hline \multirow[b]{2}{*}{ Volume $^{\mathrm{a}}$} & \multicolumn{3}{|c|}{ Perturbation } & \multicolumn{2}{|c|}{ MC } & \multirow{2}{*}{$\begin{array}{c}\text { Gaussian } \\
P_{\text {fail }}(\%)\end{array}$} & \multirow{2}{*}{$\begin{array}{c}\text { Gumbel } \\
P_{\text {fail }}(\%)\end{array}$} & \multirow{2}{*}{$\frac{\mathrm{MC}}{P_{\text {fail }}(\%)}$} \\
\hline & $\beta_{t}$ & $\mu_{c}$ & $\sigma_{c}$ & $\mu_{c}$ & $\sigma_{c}$ & & & \\
\hline 1.09 & 0.50 & 3.44 & 0.23 & 3.45 & 0.25 & 37.08 & 30.80 & 34.50 \\
\hline 1.20 & 1.46 & 3.20 & 0.21 & 3.20 & 0.22 & 5.74 & 7.16 & 8.20 \\
\hline 1.27 & 2.09 & 3.02 & 0.19 & 3.02 & 0.20 & 0.39 & 1.81 & 1.86 \\
\hline 1.31 & 2.29 & 2.97 & 0.18 & 2.97 & 0.19 & 0.11 & 1.10 & 1.20 \\
\hline 1.35 & 2.66 & 2.86 & 0.17 & 2.86 & 0.18 & 0.00 & 0.39 & 0.35 \\
\hline 1.41 & 3.01 & 2.76 & 0.16 & 2.76 & 0.17 & 0.00 & 0.13 & 0.11 \\
\hline
\end{tabular}

a Normalized volume.

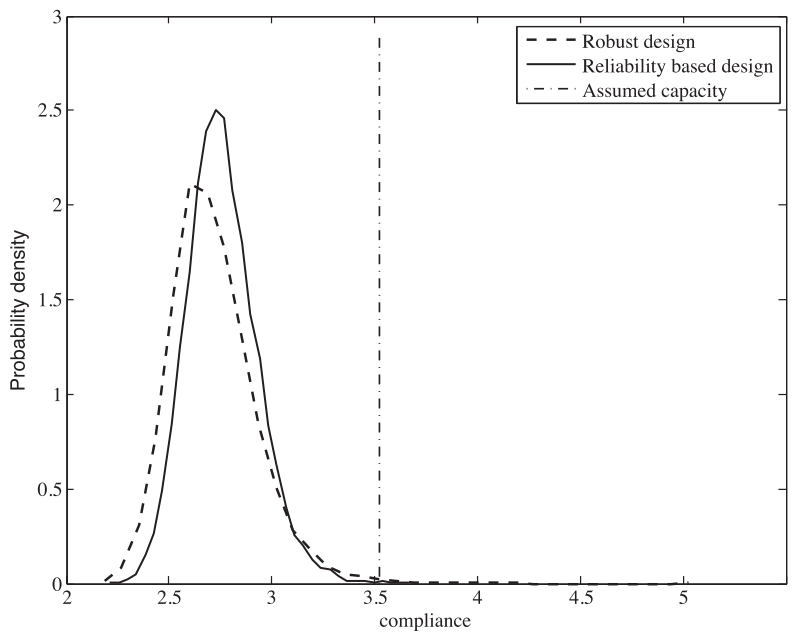

Fig. 5. Comparison of performance of RTO (expected value only) and RBTO for cantilever truss.

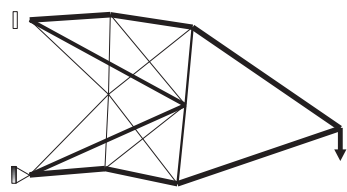

(a)

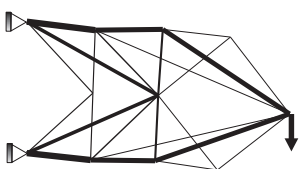

(b)
Fig. 6. Modes of failure for the RTO (expected value only) and RBTO for cantilever

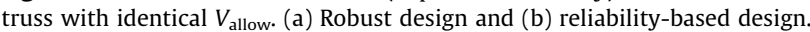

of compliance $\left(\mu_{c}=2.71\right)$ but larger standard deviation $\left(\sigma_{c}=0.19\right)$ than the RBTO solution. This leads to a probability of failure of $P[F]=0.25 \%$, nearly double that of the corresponding RBTO solution. It is noted that including multiple standard deviation of response in the RTO formulation, as in [32], would reduce this gap.

Another way to compare the two designs is to look at the failure modes, which are shown in Fig. 6. We see that the excessive deformation in the compressive member has been mitigated by the RBTO approach through the introduction of additional paths.

\subsubsection{Simply supported truss}

We now consider the simply supported ground structure shown in Fig. 7. The dimensions are $L=90$ and $H=30$ and the truss is subjected to three point loads with a common magnitude of 0.1 . We consider 6\% randomness in node location (with respect to mean spacing in horizontal direction) and choose a compliance capacity of $c^{*}=4.6$.

Fig. 8 displays the optimal topology for two target reliabilities. We plot the empirical, normal and Gumbel estimates for probabilities of failure for both designs in Fig. 9. It is interesting to observe

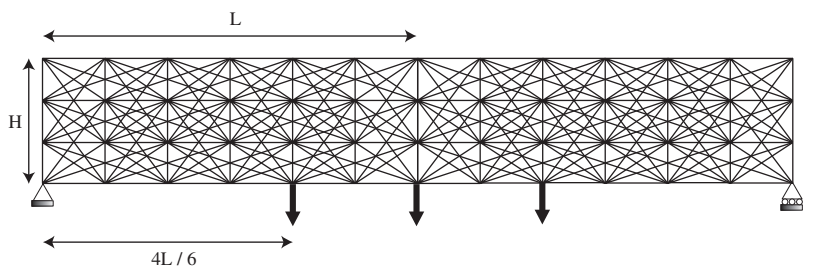

Fig. 7. Ground structure for simply supported truss.

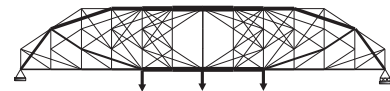

(a)

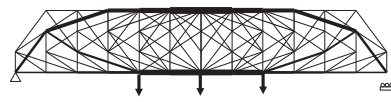

(b)
Fig. 8. Designs for simply supported truss at two levels of target reliability. (a) $\beta_{t}$ $=1.00$ and $(\mathrm{b}) \beta_{t}=3.01$

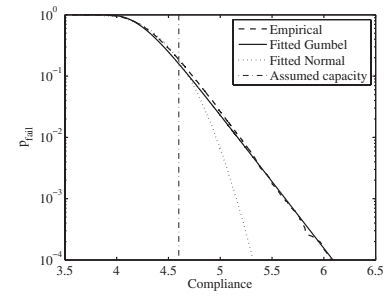

(a)

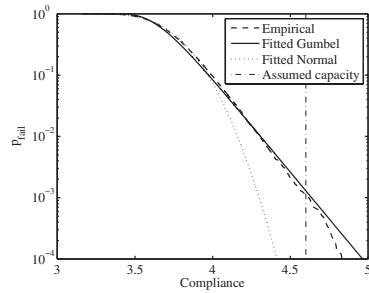

(b)
Fig. 9. Probability of failure plots with respect to $c^{*}$ for designs in Fig. 8. (a) Design in Fig. 8(a), and (b) design in Fig. 8(b).

that both normal and Gumbel estimates ( $P_{\text {fail }}^{\text {Normal }}=47.20 \%$ - and $P_{\text {fail }}^{\text {Gumbel }}=16.66 \%$ ) are close to the Monte Carlo estimate $\left(P_{\text {fail }}^{\mathrm{MC}}=48.73 \%\right)$ for the design in Fig. 8 (a), but the Gumbel distribution offers a significantly better estimate for lower probabilities of failure $\left(P_{\text {fail }}^{\text {Gumbel }}=P_{\text {fail }}^{\mathrm{MC}}=0.13 \%\right)$ for the design in Fig. 8 (b).

\subsection{Material properties}

We now demonstrate the algorithm considering uncertainty in Young's modulus. It is noted that the second derivatives of the stiffness matrix are zero for material property uncertainty, so that the last term in (13) can be dropped. Basic variables (Young's modulus of truss members) are assumed correlated. To obtain a stationery correlation field we use the following exponential decay correlation function:

$\left.\sigma_{i j}=\leftarrow \sqrt{\sigma_{i i} \sigma_{j j}} \notin \operatorname{xp} \frac{\left\|\bar{d}_{i}-\bar{d}_{j}\right\|}{L_{\exp }}\right)($

where $\sigma_{i j}$ is the correlation between element $i$ and $j, \sigma_{i i}=\sigma_{j j}$ is the variance, $\bar{d}_{i}$ is the coordinate for center of element $i$, and $L_{\exp }$ is the correlation length. The larger the correlation length the stronger the correlation. 


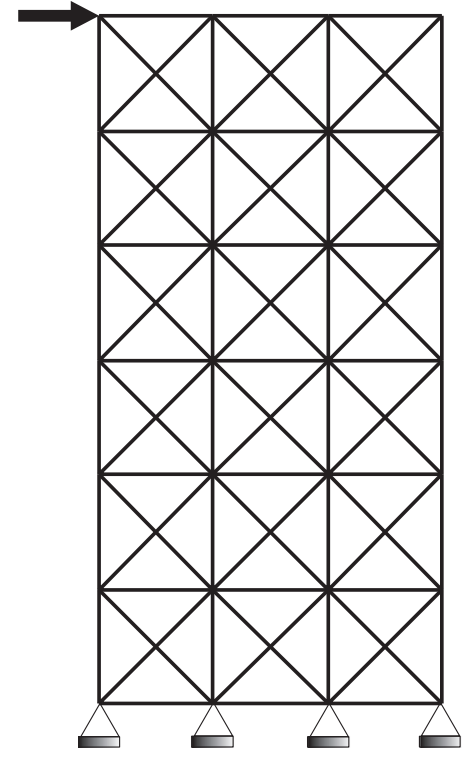

Fig. 10. Ground structure for the asymmetrically loaded cantilever truss.

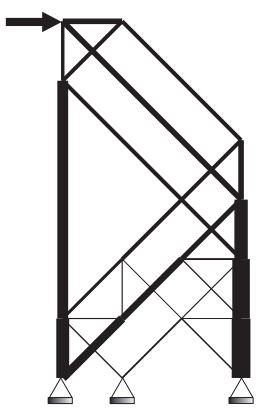

(a)

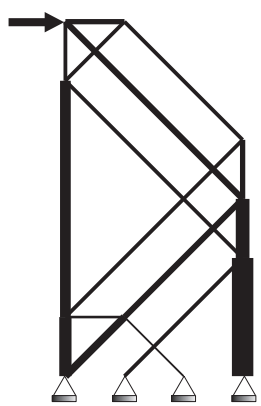

(b)

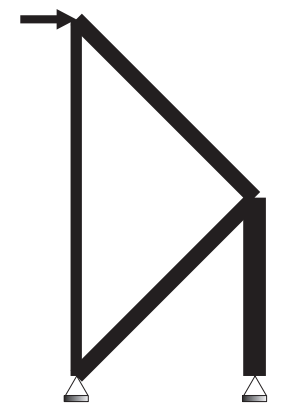

(c)
Fig. 11. Designs for material uncertainty for the asymmetrically loaded cantilever truss and $\beta_{t}=3.01$ with various levels of correlation. (a) Uncorrelated, (b) $L_{\exp }=1$ and (c) $L_{\exp }=10$.

\subsubsection{Asymmetrically loaded cantilever truss}

We conclude the example section with the cantilever truss shown in Fig. 10, previously studied in Jalalpour et al. [33]. Domain dimensions are $60 \times 30$, load magnitude is 1 and $c^{*}=20$. We choose $\sigma_{E}=0.2$, and design for a target reliability of $\beta_{t}=3.01$. Fig. 11 shows results for uncorrelated and two correlation lengths.

It is interesting to note that the design in Fig. 11 (c) is the same as deterministic design despite considering randomness in material properties. This is in line with previous findings [31] where a higher correlation length would lead to less complexity in structural design. However, it should be noted that this design requires significantly higher volume of material in comparison to the deterministic case. This can be observed in Fig. 12, which plots normalized volume of the structural system against correlation length. The curve for the normalized weight has a discontinuity at $L_{\exp }$ $=3$; this is due to the fact that the topology of the optimized design undergoes a very distinct and abrupt change as the correlation length exceeds 3.

The plot of empirical and fitted distributions for correlated basic variables design is shown in Fig. 13. This figure again shows the accuracy of Gumbel fit. The estimated $P_{\text {fail }}^{\text {Gumbel }}=4 \%$-is in agreement with Monte Carlo result $P_{\text {fail }}^{\mathrm{MC}}=4.8 \%$.

It can be observed that the design for uncorrelated basic variables in Fig. 11 (a) is more complex than the case of correlated ran-

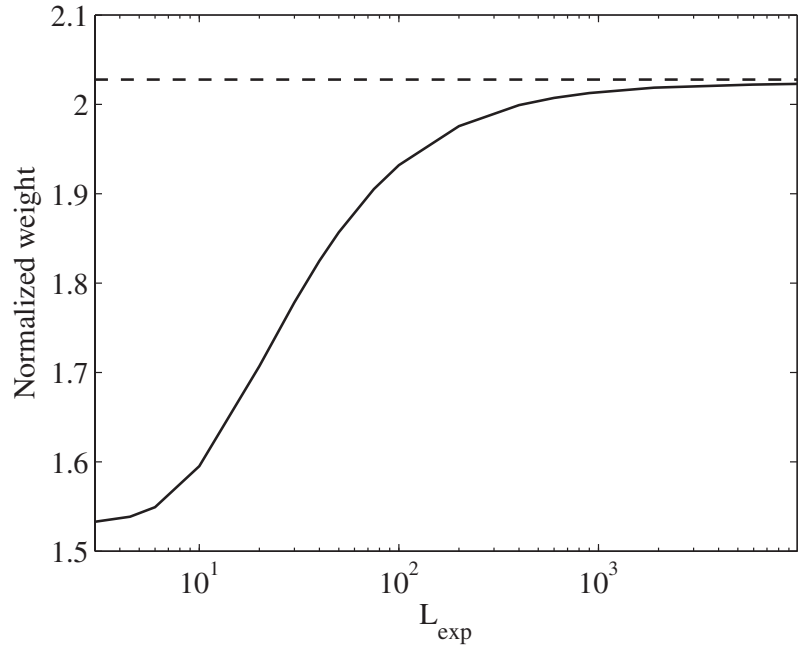

Fig. 12. Normalized weight versus correlation length for the asymmetrically loaded cantilever truss.

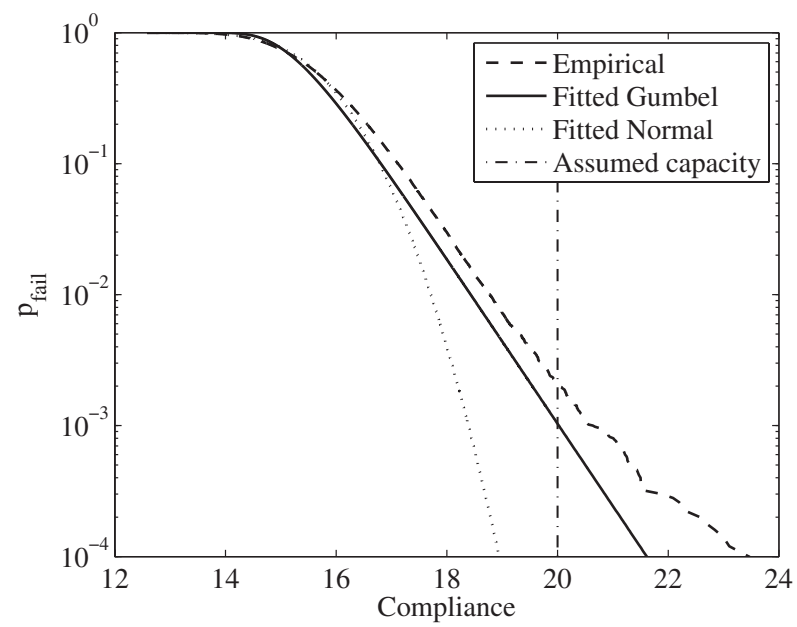

Fig. 13. Probability of failure plot with respect to $c^{*}$ for the design in Fig. 11(b)

dom variables $(b, c)$. As we discussed in previous examples, the design is also dependent on the target reliability. Therefore, we now investigate this effect, and present two designs for lower target reliabilities in Fig. 14. It is interesting that the design for $\beta_{t}=0.51$ is again similar to the deterministic design. This is in contrast to considering geometric uncertainty, which consistently leads to increased bracing throughout the structure. This is consistent with previous findings [30,32,33]. The primary reason for this phenomenon is that consideration of material uncertainty leads to $\mathbf{K}_{0, i j}=0$ as shown with Eq. (16), and that the matrices $\mathbf{K}_{0, j}$ have different structures when considering material property versus geometric uncertainties which is discussed in detail in [32].

\section{Concluding remarks}

In this article, a reliability-based topology optimization algorithm has been proposed for truss structural systems with geometric and/or material Young's modulus uncertainties. These uncertainties may result from manufacturing flaws or damage, and are assumed to be relatively small and normally distributed. Under such conditions a Gumbel probability model is used to estimate structural reliability using only first- and second-moments of 


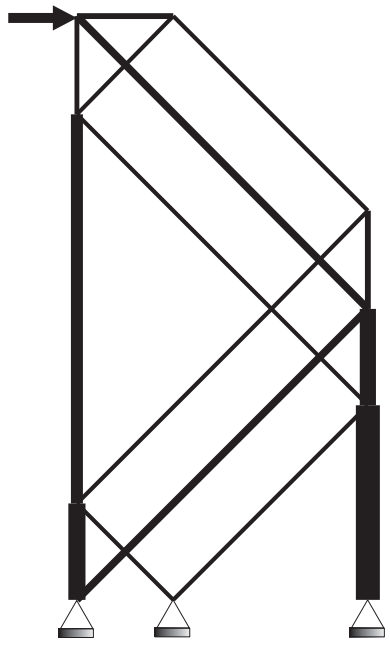

(a)

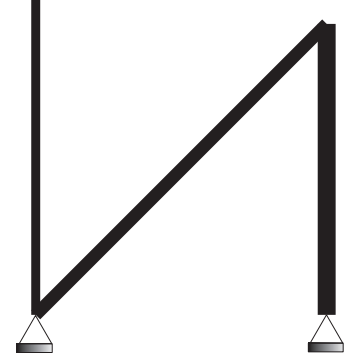

(b)
Fig. 14. Design for uncorrelated material uncertainty for the asymmetrically loaded cantilever truss and two levels of target reliability. (a) $\beta_{t}=2.34$ and (b) $\beta_{t}=0.51$.

the response, estimated using stochastic perturbation. The accuracy of the perturbation-based estimates of first- and second-moments and the Gumbel estimate of the structural reliability is verified with Monte Carlo simulation for several structures. It is observed that the estimates are acceptable without significant loss of accuracy, even for probabilities of failure as low as $0.1 \%$. Therefore, a computationally efficient single-loop RBTO algorithm without a search for the MPP is proposed.

The computational efficiency of the algorithm as compared to Monte Carlo simulation and MPP methods makes it attractive particulary in iterative structural topology optimization. The limitation of the approach are that the Gumbel model is based on the assumption of normally distributed basic variables, and may lose accuracy for very simple designs with few nodes. Regardless, under such conditions the preliminary design obtained from the proposed algorithm can be verified using more computationally intensive MPP-based reliability methods.

The field of topology optimization is currently undergoing rapid growth and finding use in structural engineering design, in addition to a wide-range of other engineering applications. It is becoming common knowledge, however, that ultra-efficient topologyoptimized solutions found under deterministic design conditions may be susceptible to uncertainties in stiffness, such as due to manufacturing defects, thus necessitating formal treatment of such uncertainties in the design problem formulation and algorithm. This work provides an efficient first step towards a reliabilitybased topology optimization under such conditions.

\section{Acknowledgements}

This work was supported by National Science Foundation under Grant No. CMMI-0928613 with Dr. Christina Bloebaum serving as program officer. This support is gratefully acknowledged.

\section{Appendix A. Sensitivities}

In the following we use direct differentiation to derive the sensitivity of the variance of compliance with respect to the design variables for use with gradient-based optimizers. The reader is referred to Jalalpour et al. [33] for the sensitivity of expected value of compliance.
To begin, we repeat the expression for variance when $\mathbf{l}=\mathbf{f}$ in Eq. (2) for convenience:

$$
\begin{aligned}
& \operatorname{var}[c]=\operatorname{tr}\left\{\mathbf{f}^{T} \mathbf{U} \mathbf{C}_{0} \mathbf{U}^{T} \mathbf{f}\right\} \\
& +2 \operatorname{tr}\left\{\left(\left(\mathbf{U}^{T} \mathbf{K}_{0} \mathbf{U}-\frac{1}{2} \mathbf{e}_{i} \mathbf{d}_{0}^{T} \mathbf{K}_{0, i j} \mathbf{d}_{0} \mathbf{e}_{j}^{T}\right) \mathbf{C}_{0}\right)^{2}\right\}
\end{aligned}
$$

Using chain rule results in:

$$
\begin{aligned}
& (\operatorname{var}[c])^{\prime}=\operatorname{tr}\left\{\mathbf{f}^{T} \mathbf{U}^{\prime} \mathbf{C}_{0} \mathbf{U}^{T} \mathbf{f}\right\} \\
& +4 \operatorname{tr}\left\{\left(\mathbf{U}^{T} \mathbf{K}_{0}^{\prime} \mathbf{U}+2 \mathbf{U}^{T} \mathbf{K}_{0} \mathbf{U}^{\prime \leftarrow}-\frac{1}{2} \mathbf{e}_{i}\left(\mathbf{d}_{0}^{T} \mathbf{K}_{0, i j}^{\prime \leftarrow} \mathbf{d}_{0}\right.\right.\right. \\
& \left.\left.\left.+2 \mathbf{d}_{0}^{T} \mathbf{K}_{0, i j} \mathbf{d}_{0}^{\prime \leftarrow}\right) \mathbf{e}_{j}^{T}\right) \mathbf{C}_{0} \mathbf{K}_{U} \mathbf{C}_{0}\right\}
\end{aligned}
$$

where primes denote the gradient with respect to the design variable (cross sectional areas for trusses). Differentiating Eqs. (9) and (11) and solving yields:

$\mathbf{U}^{\prime \leftarrow}=\mathbf{K}_{0}^{-1}\left(\mathbf{K}_{0}^{\prime} \mathbf{K}_{0}^{-1} \mathbf{K}_{0, i}-\mathbf{K}_{0, i}^{\prime \leftarrow}+\mathbf{K}_{0, i} \mathbf{K}_{0}^{-1} \mathbf{K}_{0}^{\prime \leftarrow}\right) \mathbf{d}_{0} \mathbf{e}_{i}$

$\mathbf{d}_{0}^{\prime \leftarrow}=-\mathbf{K}_{0}^{-1} \mathbf{K}_{0}^{\prime \leftarrow} \mathbf{d}_{0}$

Eq. (A.3) can be simplified to the following:

$\mathbf{U}^{\prime \leftarrow}=\left\langle\mathbf{K}_{0}^{-1}\left(-\mathbf{K}_{0}^{\prime \leftarrow} \mathbf{U}-\left(\mathbf{K}_{0, i}^{\prime \leftarrow} \mathbf{d}_{0}+\mathbf{K}_{0, i} \mathbf{d}_{0}^{\prime \leftarrow}\right) \mathbf{e}_{i}^{T}\right) \leftarrow\right.$

Substituting (A.5) into and taking advantage of symmetric matrices gives the final form of sensitivity as:

$$
\begin{aligned}
& (\operatorname{var}[c])^{\prime \leftarrow}=2 \operatorname{tr}\left\{\mathbf{d}_{0}^{T}\left(-\mathbf{K}_{0}^{\prime \leftarrow} \mathbf{U}+\left(-\mathbf{K}_{0, i}^{\leftarrow} \mathbf{d}_{0}+\mathbf{K}_{0, i} \mathbf{d}_{0}^{\prime \leftarrow}\right)\right) \mathbf{e}_{i}^{T}\right. \\
& \left.\mathbf{C}_{0} \mathbf{U}^{T} \mathbf{f}\right\}+4 \operatorname{tr}\left\{-\mathbf{U}^{T} \mathbf{K}_{0}^{\prime \leftarrow} \mathbf{U}+2 \mathbf{U}^{T}\left(\left(-\mathbf{K}_{0, i}^{\prime \leftarrow} \mathbf{d}_{0}+\mathbf{K}_{0, i} \mathbf{d}^{\prime}\right) \mathbf{e}_{i}^{T}\right) \leftarrow\right. \\
& \left.\mathbf{C}_{0} \mathbf{K}_{\mathbf{U}} \mathbf{C}_{0}\right\}-2 \operatorname{tr}\left\{\mathbf{e}_{i}\left(\mathbf{d}_{0}^{T} \mathbf{K}_{0, j}^{\leftarrow} \mathbf{d}_{0}+2 \mathbf{d}_{0}^{T} \mathbf{K}_{0, i j} \mathbf{d}_{0}^{\prime \leftarrow}\right) \mathbf{e}_{j}^{T} \mathbf{C}_{0} \mathbf{K}_{\mathbf{U}} \mathbf{C}_{0}\right\} \leftarrow
\end{aligned}
$$

Finally, the gradient of $\lambda$ in Eq. (21) can be found using:

$s^{\prime}=\frac{\sqrt{6} \phi^{\prime \prime} c}{\pi}$

$l^{\prime \leftarrow}=\mu_{c}^{\prime \leftarrow}-\nLeftarrow S^{\prime \leftarrow}$

$\lambda^{\prime \leftarrow}=\frac{-l^{\prime} \overleftarrow{s}+s^{\prime}(c l) \leftarrow}{s^{2}} \leftarrow$

where $\sigma_{c^{\prime \leftarrow}}$ and $\mu_{c^{\prime \leftarrow}}$ are gradients of the standard deviation and expected value of compliance, respectively.

\section{References}

[1] Madsen HO, Krenk S, Lind N. Methods of structural safety. Prentice-Hall Inc.; 1986.

[2] Schuëller G, Pradlwarter H, Koutsourelakis P. A critical appraisal of reliability estimation procedures for high dimensions. Probab Eng Mech 2004;19(4):463-74.

[3] Cornell C. A probability-based structural code. J Am Concr Inst 1969;66(12):974-85.

[4] Hasofer A, Lind N. An exact and invariant first-order reliability format. J Eng Mech Div 1974;100(1):111-21.

[5] Der Kiureghian A, Lin H, Hwang S. Second-order reliability approximations. J Eng Mech 1987;113(8):1208-25

[6] Hohenbichler M, Gollwitzer S, Kruse W, Rackwitz R. New light on first- and second-order reliability methods. Struct Saf 1987;4:267-84.

[7] Gasser M, Schueller G. Reliability-based optimization of structural systems Math Methods Oper Res 1997;46(12):287-307.

[8] Liu PL, Der Kiureghian A. Optimization algorithms for structural reliability. Struct Saf $1991 ; 9(3): 161-77$

[9] Karadeniz H, Toan V, Vrouwenvelder T. An integrated reliability-based design optimization of offshore towers. Reliab Eng Syst Saf 2009;94(10):1510-6.

[10] Enevoldsen I, Sørensen J. Reliability-based optimization in structural engineering. Struct Saf 1994;15(3):169-96

[11] Tu J, Choi KK, Park YH. A new study on reliability-based design optimization. J Mech Des 1999;121(4):557-64.

[12] Madsen H, Hansen P. A comparison of some algorithms for reliability based structural optimization and sensitivity analysis. In: Rackwitz R, Thoft- 
Christensen P, editors. Proceedings of the 4th IFIP WG 7.5 conference. Munich, Germany: Springer-Verlag, Berlin. p. 443451.

[13] Agarwal H. Reliability based design optimization: formulations and methodologies. Ph.D. thesis, University of Notredam; 2004

[14] Liang J, Mourelatos Z, Tu J. A single-loop method for reliability-based design optimization. ASME Conf Proc 2004;2004(46946):419-30.

[15] Kharmanda G, Lambert S, Kourdi N, Daboul A, Elhami A. Reliability-based topology optimization for different engineering applications. Int J CAD/CAM 2007;7(1):61-9.

[16] Du X, Chen W. Sequential optimization and reliability assessment method for effcient probabilistic design. J Mech Des 2005;126(2):225-33.

[17] Kang Z, Luo Y. Non-probabilistic reliability-based topology optimization of geometrically nonlinear structures using convex models. Comput Meth Appl Mech Eng 2009;198(4144):3228-38.

[18] Chiralaksanakul A, Mahadevan S. First-order approximation methods in reliabilitybased design optimization. J Mech Des 2005;127(5):851-7.

[19] Fu G, Frangopol DM. Balancing weight, system reliability and redundancy in a multiobjective optimization framework. Struct Saf 1990;7(24):165-75.

[20] Hendawi S, Frangopol DM. System reliability and redundancy in structural design and evaluation. Struct Saf 1994;16(12):47-71.

[21] Thoft-Christensen P, Dalsgård Sørensen J. Reliability of structural systems with correlated elements. Appl Math Modell 1982;6(3):171-8.

[22] Nguyen TH, Song J, Paulino GH. Single-loop system reliability-based design optimization using matrix-based system reliability method: theory and applications. J Mech Des 2010;132(1):011005.

[23] Silva M, Tortorelli D, Norato J, Ha C, Bae HR. Component and system reliabilitybased topology optimization using a single-loop method. Struct Multi Optim 2010;41:87-106.

[24] Park S, Choi S, Sikorsky C, Stubbs N. Efficient method for calculation of system reliability of a complex structure. Int J Solids Struct 2004;41(1819):5035-50.

[25] Tsompanakis Y, Lagaros N, Papadrakakis M. Structural design optimization considering uncertainties. Taylor and Francis; 2008.

[26] Schueller G, Jensen H. Computational methods in optimization considering uncertainties - an overview. Comput Meth Appl Mech Eng 2008;198(1):2-13.

[27] Igusa T, Der Kiureghian A. Response of uncertain systems to stochastic excitation. J Eng Mech 1988;114(5):812-32.

[28] Stockl G. Topology optimization of trusses under stochastic uncertainty. J Appl Math Mech 2001;81(S3):697-8.

[29] Maute K, Frangopol DM. Reliability-based design of mems mechanisms by topology optimization. Comput Struct 2003;81(8-11):813-24.
[30] Guest J, Igusa T. Structural optimization under uncertain loads and nodal locations. Comput Meth Appl Mech Eng 2008;198(1):116-24.

[31] Asadpoure A, Guest J, Igusa T. Structural topology optimization considering correlated uncertainties in elastic modulus. Collection of Technical Papers AIAA/ASME/ASCE/AHS/ASC Structures, Structural Dynamics and Materials Conference, 2010.

[32] Asadpoure A, Tootkaboni M, Guest JK. Robust topology optimization of structures with uncertainties in stiffness: application to truss structures. Comput Struct 2011;89(1112):1131-41.

[33] Jalalpour M, Igusa T, Guest J. Optimal design of trusses with geometric imperfections: accounting for global instability. Int J Solids Struct 2011;48(21):3011-9.

[34] Chen S, Chen W, Lee S. Level set based robust shape and topology optimization under random field uncertainities. Struct Multidiscipl. Optim., 2010:507-24.

[35] Schevenels M, Lazarov BS, Sigmund O. Robust topology optimization accounting for spatially varying manufacturing errors. Comput Meth Appl Mech Eng 2011;200:3613-27.

[36] Tootkaboni M, Asadpoure A, Guest JK. Topology optimization of continuum structures under uncertainty: a polynomial chaos approach. Comput Meth Appl Mech Eng 2012;201:204(0):263-75.

[37] Ditlevsen O, Madsen H. Structural reliability methods 2007. Available from: $<$ http://www.web.mek.dtu.dk/staff/od/books.htm>.

[38] Liu P, Der Kiureghian A. Multivariate distribution models with prescribed marginals and covariances. Probab Eng Mech 1986;1(2):105-12.

[39] Gumbel EJ. Statistical theory of extreme values and some practical applications; a series of lectures. Washington: U.S. Govt. Print. Office; 1954.

[40] Ditlevsen O. Generalized second moment reliability index. J Struct Mech 1979;7(4):435-51.

[41] The MathWorks Inc. MATLAB - optimization toolbox, version 6.2. Natick, Massachusetts: The MathWorks Inc; 2012. http://www.mathworks.com/ products/optimization/.

[42] Kirsch U. Optimal topologies of truss structures. Comput Meth Appl Mech Eng 1989;72(1):15-28.

[43] Rozvany GIN, BM P, Kirsch U. Layout optimization of structures. Appl Mech Rev 1995;48(2):41-119.

[44] Bendsøe MP, Sigmund O. Topology optimization: theory, methods and applications. Springer; 2003.

[45] Mogami K, Nishiwaki S, Izui K, Yoshimura M, Kogiso N. Reliabilitybased structural optimization of frame structures for multiple failure criteria using topology optimization techniques. Struct Multi Optim 2006;32:299-311. 